\title{
Spherical Harmonic Analyses for the Spheroidal Earth
}

\author{
By A.B. KAHLE, J.W. KeRn and E.H. Vestine \\ The RAND Corporation, Santa Monica, California \\ (Received November 25, 1964)
}

\begin{abstract}
Most spherical harmonic analyses of the surface geomagnetic field assume that the earth is a sphere. Two methods are devised for correcting the coefficients derived from such analyses for the oblateness of the earth's surface. The corrections required are shown to be significant. The largest, the change in the $g_{3}{ }^{0}$ coefficients, is about $80 \gamma$. The appearance of an erroneous unipole term in spherical harmonic representations of the geomagnetic field is discussed. The calculation of such a term is shown to yield information regarding absolute errors in geomagnetic data.
\end{abstract}

\section{Introduction}

The magnetic field of the earth is usually represented mathematically by a series of solid spherical harmonics. The analysis is based on the approximation that the earth is a perfect sphere. The earth is, however, more nearly a spheroid, the polar and equatorial radii differing by over 20 kilometers. At the end of the nineteenth century, Schmidt (Chapman and Bartels, 1940) showed that the corrections necessary to take into account the difference in shape are quite small. As a result of Schmidt's analysis, the effect has long been ignored as being too small to be significant. Considering the errors of measurement and limited knowledge of the field at the earth's surface in the past, this viewpoint was justifiable. This was particularly true since harmonic analyses that neglect the earth's ellipticity do adequately fit the field on the surface.

However, the coefficients for the harmonic series derived from such analyses are, necessarily, only approximations to the desired solid spherical harmonic coefficients. Only these solid spherical harmonic representations can correctly be used for extrapolation of the field throughout source-free regions. Such extrapolations have become of importance in connection with studies of both the geomagnetic field in space and in the earth's interior. Since world magnetic surveys are now being made aboard earth satellites, which intend to determine the field to within $10 \gamma$ (where $1 \gamma=10^{-5}$ gauss), the effect of the earth's oblateness warrants closer study. Cain (1963) has recently pointed this out. We have devised an approximate method correcting the coefficients of existing spherical analyses for the change in radius with latitude due to the earth's oblateness. An additional correction due to the small difference in geodetic and geographic latitude will be reported on later.

\section{Derivation of Potential Over a Spheroidal Earth}

In the usual analysis of the earth's magnetic field, the potential due to internal sources is expressed as 


$$
V=a \sum_{n=1}^{\infty} \sum_{m=0}^{n}\left(\frac{a}{r}\right)^{n+1}\left(g_{n}{ }^{m} \cos m \lambda+h_{n}{ }^{m} \sin m \lambda\right) P_{n}{ }^{m}(\theta),
$$

where $a$ is the radius of a reference sphere, $\lambda$ the east longitude and $\theta$ the colatitude. These coefficients, the $g$ 's and $h^{\prime}$ s are calculated assuming the surface of the earth to be a sphere of radius $r=a$, and do indeed fit the observed data over the surface. However, because of the earth's nonspherical shape, these coefficients are not the appropriate solid spherical harmonics to use when attempting to extrapolate the field inward or outward.

The correct potential, expressed in terms of properly defined solid spherical harmonics, referred to a sphere of constant radius $a$ is

$$
V=a \sum_{n=1}^{\infty} \sum_{m=0}^{n}\left(\frac{a}{r}\right)^{n+1}\left(G_{n}{ }^{m} \cos m \lambda+H_{n}{ }^{m} \sin m \lambda\right) P_{n}{ }^{m}(\theta)
$$

where the radial distance to the earth's surface is now a function of colatitude,

$$
r=\frac{\alpha}{\left(1+\varepsilon^{2} \cos ^{2} \theta\right)^{1 / 2}},
$$

with $\alpha$ the equatorial radius, and $\varepsilon^{2}=\left(\alpha^{2}-\beta^{2}\right) / \beta^{2}, \beta$ the polar radius.

A method is outlined here to find the proper values of $G$ and of $H$ to define this potential, from the now existing $g$ and $h$ that are derived on the assumption that the earth is spherical.

On the earth's surface, the two expressions (Eqs. 1 and 2) for the potential are equal. This provides the equation

$$
\begin{aligned}
a & \sum_{n=1}^{\infty} \sum_{m=0}^{n}\left(g_{n}{ }^{m} \cos m \lambda+h_{n}{ }^{m} \sin m \lambda\right) P_{n}{ }^{m}(\theta) \\
& =a \sum_{n=1}^{\infty} \sum_{m=0}^{n}\left(\frac{a}{r}\right)^{n+1}\left(G_{n}{ }^{m} \cos m \lambda+H_{n}{ }^{m} \sin m \lambda\right) P_{n}{ }^{m}(\theta) .
\end{aligned}
$$

We note that

$$
\begin{aligned}
& \left(\frac{a}{r}\right)^{n+1} P_{n}^{m}(\theta)=\left(\frac{a}{\alpha}\right)^{n+1}\left(1+\varepsilon^{2} \cos ^{2} \theta\right)^{\frac{n+1}{2}} P_{n}^{m}(\theta) \\
& \cong\left(\frac{a}{\alpha}\right)^{n+1}\left(1+\frac{n+1}{2} \varepsilon^{2} \cos ^{2} \theta\right) P_{n}^{m}(\theta)
\end{aligned}
$$

since $\varepsilon^{2}$ is small $(<.01)$. Also

$$
\cos \theta P_{n}^{m}(\theta)=\frac{1}{2 n+1}\left\{\left[(n+1)^{2}-m^{2}\right]^{1 / 2} P_{n+1}^{m}+\left[n^{2}-m^{2}\right]^{1 / 2} P_{n-1}^{m}\right\},
$$

(Chapman and Bartels, 1940).

Therefore

$$
\cos ^{2} \theta P_{n}^{m}(\theta)=J_{n}{ }^{m} P_{n-2}^{m}+K_{n}^{m} P_{n}{ }^{m}+L_{n}{ }^{m} P_{n+2}^{m},
$$

where

$$
J_{n}{ }^{m}=\frac{\left[n^{2}-m^{2}\right]^{1 / 2}\left[(n-1)^{2}-m^{2}\right]^{1 / 2}}{(2 n+1)(2 n-1)}
$$




$$
\begin{aligned}
K_{n}{ }^{m} & =\frac{\left[(n+1)^{2}-m^{2}\right]}{(2 n+1)(2 n+3)}+\frac{\left[n^{2}-m^{2}\right]}{(2 n+1)(2 n-1)} \\
L_{n}{ }^{m} & =\frac{\left[(n+1)^{2}-m^{2}\right]^{1 / 2}\left[(n+2)^{2}-m^{2}\right]^{1 / 2}}{(2 n+1)(2 n+3)} .
\end{aligned}
$$

Thus

$$
\begin{aligned}
& \left(\frac{a}{r}\right)^{n+1} P_{n}{ }^{m}(\theta) \cong\left(\frac{a}{\alpha}\right)^{n+1}\left[\left(\frac{n+1}{2} \varepsilon^{2} J_{n}{ }^{m}\right) P^{m}{ }_{n-2}\right. \\
& \left.+\left(1+\frac{n+1}{2} \varepsilon^{2} K_{n}{ }^{m}\right) P_{n}{ }^{m}+\left(\frac{n+1}{2} \varepsilon^{2} L_{n}{ }^{m}\right) P^{m}{ }_{n+2}\right] .
\end{aligned}
$$

By substituting Eq. (4) into Eq. (3) and equating like terms in $P_{n}{ }^{m}(\theta)$, we find

where

$$
g_{n}{ }^{m} \cong G_{n-2}^{m} L_{n-2}^{m \prime}+G_{n}{ }^{m} K_{n}{ }^{m \prime}+G_{n+2}^{m} J_{n+2}^{m \prime} \text {, }
$$

$$
\begin{aligned}
J_{n}{ }^{m \prime} & =\left(\frac{a}{\alpha}\right)^{n+1} \frac{n+1}{2} \varepsilon^{2} J_{n}{ }^{m} \\
K_{n}{ }^{m \prime} & =\left(\frac{a}{\alpha}\right)^{n+1}\left(1+\frac{n+1}{2} \varepsilon^{2} K_{n}{ }^{m}\right) \\
L_{n}{ }^{m \prime} & =\left(\frac{a}{\alpha}\right)^{n+1} \frac{n+1}{2} \varepsilon^{2} L_{n}{ }^{m} .
\end{aligned}
$$

Since $\varepsilon^{2}$ is small, and $K_{n}{ }^{m}$ is bounded by 0.6 , the constant $K_{n}{ }^{m \prime}$ is nearly unity, while $L_{n}{ }^{m \prime}$ and $J_{n}{ }^{m \prime}$ are much smaller. Solving Eq. (5) for $G_{n}{ }^{m}$, substituting the like approximations for $G_{n-2}^{m}$ and $G_{n+2}^{m}$, and ignoring second order terms, we find

$$
G_{n}{ }^{m} \cong \frac{g_{n}{ }^{m}}{K_{n}{ }^{m \prime}}-\frac{L_{n-2}{ }^{m \prime}}{K_{n-2}{ }^{m \prime}} g^{m}{ }_{n-2}-\frac{J^{m \prime}{ }_{n+2}}{K^{m \prime}{ }_{n+2}} g^{m+2}{ }_{n+2} .
$$

The $H_{n}{ }^{m}$ equation is similar. Thus we have found equations to determine the desired coefficients.

A second method for calculating $G_{n}{ }^{m}$ and $H_{n}{ }^{m}$ was constructed by assuming that the error in extrapolation from $r$ to $a$ that results from using the incorrect coefficients is negligible. On this basis, we can rewrite Eq. (3) in the form

$$
\begin{gathered}
a \sum_{n=1}^{\infty} \sum_{m=0}^{n}\left(\frac{r}{a}\right)^{n+1}\left(g_{n}{ }^{m} \cos m \lambda+h_{n}{ }^{m} \sin m \lambda\right) P_{n}{ }^{m}(\theta) \\
\cong a \sum_{n=1}^{\infty} \sum_{m=0}^{n}\left(G_{n}{ }^{m} \cos m \lambda+H_{n}{ }^{m} \sin m \lambda\right) P_{n}{ }^{m}(\theta) .
\end{gathered}
$$

Then proceeding as before, but without having to invert as in Eq. (5),

$$
G_{n}{ }^{m} \cong L_{n-2}^{m \prime \prime} g_{n-2}^{m}+K_{n}^{m \prime \prime} g_{n}{ }^{m}+J_{n+2}^{m \prime \prime} g_{n+2}^{m},
$$

where

$$
\begin{aligned}
& J_{n}{ }^{m \prime \prime}=\left(\frac{\alpha}{a}\right)^{n+1}\left(-\frac{n+1}{2} \varepsilon^{2} J_{n}{ }^{m}\right) \\
& K_{n}{ }^{m \prime \prime}=\left(\frac{\alpha}{a}\right)^{n+1}\left(1-\frac{n+1}{2} \varepsilon^{2} K_{n}{ }^{m}\right) \\
& L_{n}{ }^{m \prime \prime}=\left(\frac{\alpha}{a}\right)^{n+1}\left(-\frac{n+1}{2} \varepsilon^{2} L_{n}{ }^{m}\right) .
\end{aligned}
$$


These double-prime constants differ from the single-prime constants of Eq. (6) only in second order terms in $\varepsilon^{2}$.

\section{Test Field}

A test field was constructed. Coefficients of the same order of magnitude as those for the earth's field were taken to be the values of $G$ and of $H$ that give a field correct on the sphere of mean radius $a$. This field was extrapolated by the conventional spherical harmonic method to the oblate spheroid representing the earth's surface, with values of the potential calculated for every 5 degrees of latitude and longitude. These values were then subjected to harmonic analysis in the same manner as is the earth's field, i.e., assuming them to lie on a spherical surface. The new coefficients derived from this analysis then represent the values of $g$ and of $h$. Upon applying Eqs. (6) and (7), the original $G$ and $H$ were recovered to within a very small error. The original $G$, the magnitude of the difference between these and the $g$, and the small difference between the original $G$ are listed in Table 1 along with the recalculated $G$. The results for the $H$ are similar. As can be seen, the errors are small,

Table 1 Test Field Calculations, in units of $10^{-6} \mathrm{cgs}$

\begin{tabular}{|c|c|c|c|c|}
\hline \multirow{3}{*}{$n, m$} & \multirow{2}{*}{$\begin{array}{l}\text { Original Field } \\
\text { on Sphere }\end{array}$} & \multirow{2}{*}{$\begin{array}{l}\text { Extrapolated } \\
\text { to Spheroid }\end{array}$} & \multicolumn{2}{|c|}{ Calculated on Sphere } \\
\hline & & & Method I & Method II \\
\hline & $G^{m}{ }_{n}$ & $\left|G^{m}{ }_{n}-g^{m}{ }_{n}\right|$ & $\left|\Delta G_{n}^{m}\right|$ & $\left|\Delta G_{n}^{m}\right|$ \\
\hline 0 & 0 & 13 & 0.4 & 0.5 \\
\hline 1 & $\begin{array}{l}-300000 \\
-20000\end{array}$ & $\begin{array}{r}533 \\
2\end{array}$ & 2.3 & $\begin{array}{l}7.0 \\
0.4\end{array}$ \\
\hline 2 & $\begin{array}{r}-10000 \\
30000 \\
10000\end{array}$ & $\begin{array}{r}5 \\
49 \\
10\end{array}$ & $\begin{array}{l}0.3 \\
0.3 \\
0.0\end{array}$ & $\begin{array}{l}0.4 \\
1.1 \\
0.1\end{array}$ \\
\hline 3 & $\begin{array}{r}10000 \\
-10000 \\
10000 \\
10000\end{array}$ & $\begin{array}{r}790 \\
52 \\
8 \\
29\end{array}$ & $\begin{array}{l}1.8 \\
0.3 \\
0.3 \\
0.2\end{array}$ & $\begin{array}{l}6.5 \\
0.1 \\
0.0 \\
0.3\end{array}$ \\
\hline 4 & $\begin{array}{r}8000 \\
6000 \\
4000 \\
-\quad 4000 \\
3000\end{array}$ & $\begin{array}{r}10 \\
115 \\
26 \\
6 \\
13\end{array}$ & $\begin{array}{l}0.3 \\
0.5 \\
0.5 \\
0.8 \\
0.6\end{array}$ & $\begin{array}{l}0.6 \\
1.7 \\
0.2 \\
1.0 \\
0.6\end{array}$ \\
\hline 5 & $\begin{array}{r}2000 \\
3000 \\
2000 \\
-\quad 80 \\
-\quad 2000 \\
-\quad 600\end{array}$ & $\begin{array}{r}41 \\
34 \\
39 \\
23 \\
2 \\
2\end{array}$ & $\begin{array}{l}3.2 \\
0.2 \\
0.4 \\
0.4 \\
0.3 \\
0.1\end{array}$ & $\begin{array}{l}2.8 \\
0.4 \\
0.0 \\
0.6 \\
0.3 \\
0.1\end{array}$ \\
\hline 6 & $\begin{array}{r}60 \\
-\quad 1000 \\
-\quad 500 \\
-\quad 3000 \\
-\quad 300 \\
-\quad 1000\end{array}$ & $\begin{array}{r}40 \\
34 \\
15 \\
21 \\
8 \\
1 \\
5\end{array}$ & $\begin{array}{l}0.5 \\
0.6 \\
0.1 \\
0.3 \\
0.2 \\
0.4 \\
0.4\end{array}$ & $\begin{array}{l}0.1 \\
1.2 \\
0.1 \\
0.2 \\
0.1 \\
0.4 \\
0.4\end{array}$ \\
\hline
\end{tabular}


less than one $\gamma$, compared to changes in the coefficients of up to $79 \gamma$. Equation (6) appears to give slightly better results. The $n=3, m=0$ term has the greatest effect, since it receives a contribution from the large $n=1, m=0$ term.

In these and subsequent calculations we use

$$
\begin{aligned}
& a=6371.2 \mathrm{~km} \text {, mean radius of the earth } \\
& \alpha=6378.155 \mathrm{~km} \text {, equatorial radius } \\
& \varepsilon^{2}=0.00673899, \text { ellipticity parameter. }
\end{aligned}
$$

A feature of the harmonic analysis that determines the $g_{n}{ }^{m}$ coefficients for the test field is the appearance of a $g_{0}{ }^{0}$ term. This term at first appears to represent a magnetic unipole. Such unipoles have been shown to be physically unrealizable by Maxwell. Clearly, we can require in general that the solid spherical harmonic $G_{0}{ }^{0}$ be zero, to comply with the physical requirement that no magnetic unipoles exist. Then we note that the $g_{0}{ }^{0}$ term is obtained by analyzing a potential field on a spheroid with the erroneous assumption that it is spherical.

In most analyses of the geomagnetic field, the coefficients are fitted by a least-squares method and $g_{0}{ }^{0}$ is required to be zero, or in the case of an analysis of $X$ or $Y$ or both, $g_{0}{ }^{0}$ cannot be determined and is assumed to be zero. We have already noted that in such analyses, the earth is assumed to be a sphere. It follows logically that an error is made by suppressing the $g_{0}{ }^{0}$ term. We can compute the value of the neglected $g_{0}{ }^{0}$ term by means of Eq. (6). Setting $m$ and $n$ equal to zero, and noticing that $g_{2}{ }^{0}$ is nonexistent, we have

$$
G_{0}{ }^{0}=\frac{g_{0}{ }^{0}}{K_{0}{ }^{0 \prime}}-\frac{J_{2}^{0 \prime}}{K_{2}^{0 \prime}} g_{2}{ }^{0}
$$

Setting $G_{0}{ }^{0}$ identically equal to zero and solving for $g_{0}{ }^{0}$ effectively suppresses the nonexistent unipole in the final solid spherical harmonics. Then Eq. (8) gives

$$
g_{0}{ }^{0}=\frac{K_{0}{ }^{\prime \prime} J_{2}{ }^{0 \prime}}{K_{2}{ }^{0 \prime}} g_{2}{ }^{0} .
$$

Using the defining equations for $J_{n}{ }^{m \prime}$ and $K_{n}{ }^{m \prime}$, and neglecting terms in $\varepsilon^{4}$ or higher, Eq. (9) can be reduced to the approximate equation

$$
g_{0}{ }^{0} \cong \frac{\varepsilon^{2}}{5} \frac{a}{\alpha} g_{2}{ }^{0}
$$

This equation, applied to the value of $g_{2}{ }^{0}=-9995 \times 10^{-6} \mathrm{cgs}$ for the test field, yields $g_{0}{ }^{0}$ $=-13.5 \times 10^{-6} \mathrm{cgs}$. This calculated value is to be compared with the value of $-13 \times 10^{-6} \mathrm{cgs}$ derived by the harmonic analysis. Equation (10) is clearly accurate enough for our present purposes. The value of the corresponding $h_{0}{ }^{0}$ term is, of course, identically zero.

\section{Application to the Earth's Field}

Our complete method for calculating corrected solid spherical harmonic coefficients from existing analyses of the geomagnetic field is as follows :

(1) The value of $g_{0}{ }^{0}$ that was suppressed by the analysis is recovered by applying Eq. (10). 
Table 2 Correction Applied to Various Analyses

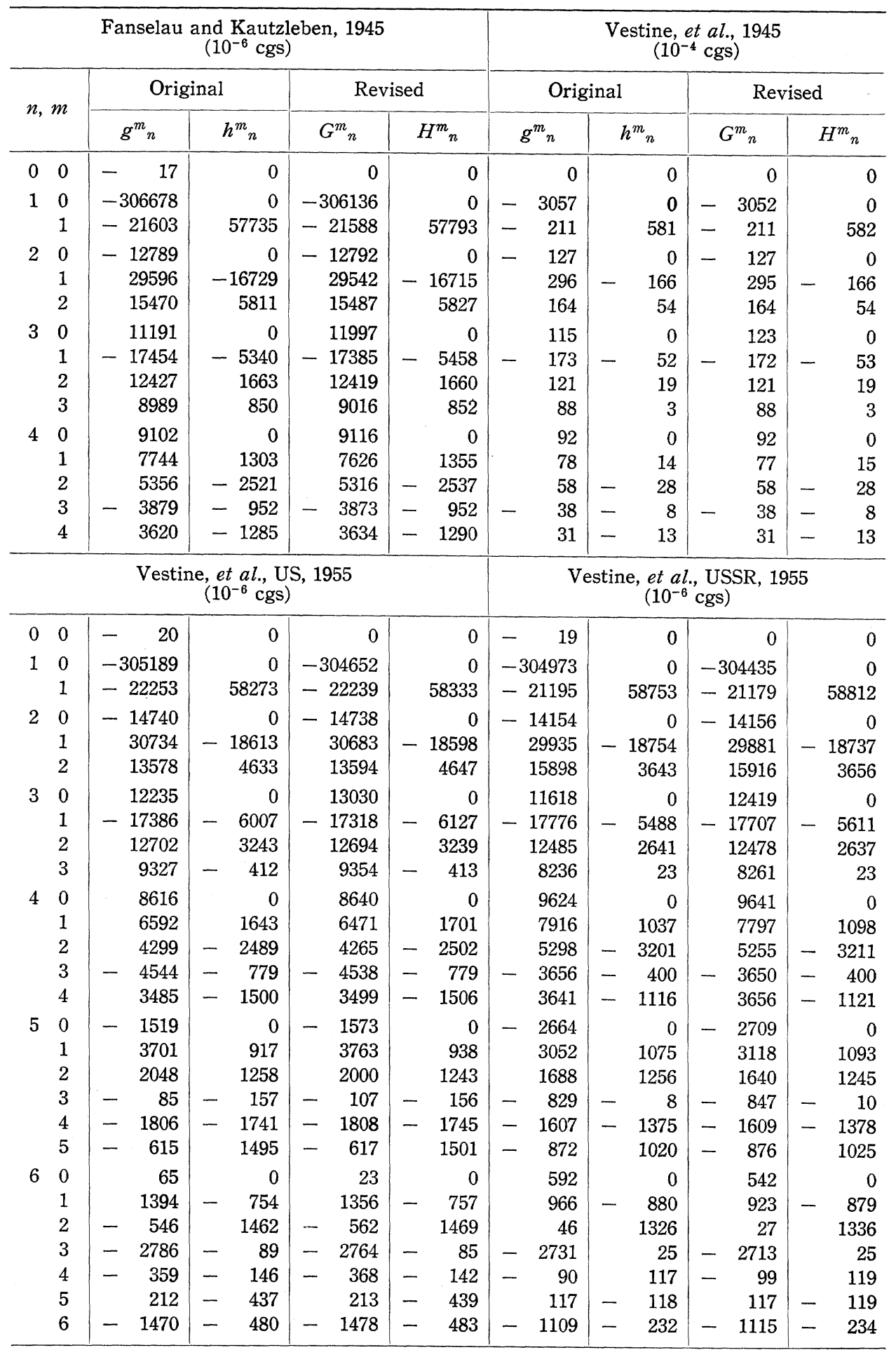


Table 2 (Continued)

\begin{tabular}{|c|c|c|c|c|c|c|c|c|c|c|c|c|c|c|c|c|c|}
\hline \multicolumn{10}{|c|}{$\begin{array}{l}\text { Finch and Leaton, } 1955 \\
\left(10^{-4} \mathrm{cgs}\right)\end{array}$} & \multicolumn{8}{|c|}{$\begin{array}{l}\text { Nagata and Oguti, } 1958.5 \\
\left(10^{-4} \mathrm{cgs}\right)\end{array}$} \\
\hline \multirow{2}{*}{\multicolumn{2}{|c|}{$n, m$}} & \multicolumn{4}{|c|}{ Original } & \multicolumn{4}{|c|}{ Revised } & \multicolumn{4}{|c|}{ Original } & \multicolumn{4}{|c|}{ Revised } \\
\hline & & \multicolumn{2}{|c|}{$g^{m}{ }_{n}$} & \multicolumn{2}{|c|}{$h_{n}^{m}$} & \multicolumn{2}{|c|}{$G_{n}^{m}$} & \multicolumn{2}{|c|}{$H^{m}{ }_{n}$} & \multicolumn{2}{|c|}{$g_{n}^{m}$} & \multicolumn{2}{|c|}{$h_{n}^{m}$} & \multicolumn{2}{|c|}{$G_{n}^{m}$} & \multicolumn{2}{|c|}{$H_{n}^{m}$} \\
\hline 0 & 0 & & 0 & & 0 & & 0 & & 0 & & 0 & & 0 & & 0 & & 0 \\
\hline 1 & 0 & - & 3055 & & 0 & - & 3050 & & 0 & - & 3045 & & 0 & - & 3040 & & 0 \\
\hline & 1 & & 227 & & 590 & - & 227 & & 591 & - & 222 & & 584 & - & 222 & & 585 \\
\hline 2 & 0 & - & 152 & & 0 & - & 152 & & 0 & - & 151 & & 0 & - & 151 & & 0 \\
\hline & 1 & & 303 & - & 190 & & 302 & - & 190 & & 295 & - & 194 & & 294 & - & 194 \\
\hline & 2 & & 158 & & 24 & & 158 & & 24 & & 149 & & 21 & & 149 & & 21 \\
\hline 3 & 0 & & 118 & & 0 & & 126 & & 0 & & 116 & & 0 & & 124 & & 0 \\
\hline & 1 & - & 191 & - & 45 & - & 190 & - & 46 & - & 186 & - & 45 & - & 185 & - & 46 \\
\hline & 2 & & 126 & & 29 & & 126 & & 29 & & 123 & & 24 & & 123 & & 24 \\
\hline & 3 & & 91 & - & 9 & & 91 & - & 9 & & 93 & - & 9 & & 93 & - & 9 \\
\hline 4 & 0 & & 95 & & 0 & & 95 & & 0 & & 94 & & 0 & & 94 & & 0 \\
\hline & 1 & & 80 & & 15 & & 79 & & 16 & & 75 & & 19 & & 74 & & 20 \\
\hline & 2 & & 58 & - & 31 & & 58 & - & 31 & & 64 & - & 23 & & 64 & - & 23 \\
\hline & 3 & - & 38 & - & 4 & - & 38 & - & 4 & - & 37 & - & 3 & - & 37 & - & 3 \\
\hline & 4 & & 31 & - & 17 & & 31 & - & 17 & & 29 & - & 20 & & 29 & - & 20 \\
\hline
\end{tabular}

Jensen and Cain, 1960 $\left(10^{-6} \mathrm{cgs}\right)$

\begin{tabular}{|c|c|c|c|c|c|}
\hline 0 & 0 & 21 & 0 & 0 & 0 \\
\hline 1 & 0 & -304112 & 0 & -303578 & 0 \\
\hline & 1 & -21474 & 57989 & -21454 & 58047 \\
\hline 2 & 0 & -16023 & 0 & -16021 & 0 \\
\hline & 1 & 29591 & - 19124 & 29536 & -19110 \\
\hline & 2 & 15451 & 1823 & 15468 & 1832 \\
\hline 3 & 0 & 12607 & 0 & 13400 & 0 \\
\hline & 1 & -20292 & - 4857 & -20219 & 4976 \\
\hline & 2 & 12857 & 2104 & 12847 & 2103 \\
\hline & 3 & 8217 & 266 & 8240 & 265 \\
\hline 4 & 0 & 9553 & 0 & 9573 & 0 \\
\hline & 1 & 8185 & 2137 & 8069 & 2190 \\
\hline & 2 & 5570 & 2557 & 5529 & 2560 \\
\hline & 3 & - 3350 & 206 & - 3346 & 207 \\
\hline & 4 & 2764 & - 1873 & 2775 & 1880 \\
\hline
\end{tabular}

(2) The corrected coefficients are calculated using either Eq. (6) or Eq. (7), preferably the former. We require the corrected coefficient $G_{0}{ }^{0}$ to be identically zero.

Corrected coefficients for the commonly used analyses of the geomagnetic field (Fanselau and Kautzleben, 1958; Vestine. et al., 1947; Vestine, et al., 1963; Jensen and Cain, 1962; Nagat a and Oguti, 1962) were calculated by application of the above procedure. The results are given in Table 2. As can be seen, the corrections are in almost all cases smaller than the differences among the various analyses. The low-degree zonal harmonic terms provide a few notable exceptions. The $g_{1}{ }^{0}$ correction varies from $-50 \gamma$ to $-54 \gamma$ for the sets of coefficients considered. For $g_{2}{ }^{0}$ (and most other terms with $n$ even), the corrections are small and of little interest. However, because of the magnitude of $g_{1}{ }^{0}$ in Eqs. (6) or (7), the correc- 
tions for $g_{3}{ }^{0}$ are from $79 \gamma$ to $81 \gamma$.

Cain (1963) has called attention to the fact that failure to use a spheroidal earth may affect extrapolations of the geomagnetic field to satellite levels by about $100 \mathrm{r}$ or more. We calculated this effect both at satellite altitudes and for extrapolations to the surface of the earth's core, the presumed internal source of the earth's magnetic field. Using the U.S. 1955 analysis at a geocentric distance $r=6871.2 \mathrm{~km}$ (about $500 \mathrm{~km}$ above ground level) some original and corrected values of $B$ in gammas were calculated. At $\theta=30^{\circ}, \lambda=0^{\circ}$, these were respectively 40310 and $40150 \gamma$, a difference of $160 \gamma$. At $\theta=30^{\circ}, \lambda=180^{\circ}$, the corresponding values were 43300 and $43110 \gamma$, a difference of $190 \gamma$. At $\theta=90^{\circ}, \lambda=180^{\circ}$, we find $B=28170 \gamma$ and $28220 \gamma$, the latter corrected value differing by $50 \gamma$. These differences, as Cain has remarked, are clearly of considerable importance in relating magnetic surveys at satellite level to ground values. On the other hand, at $r=3371.2 \mathrm{~km}$, near the surface of the earth's core, corresponding values at $\theta=30^{\circ}, \lambda=0^{\circ}$, are $274200 \gamma$ and $273800 \gamma$, respectively, and at $\theta=30^{\circ}$, $\lambda=180^{\circ}$, we find $414800 \gamma$ and $409600 \gamma$. However, these differences (amounting to about $1 \%$ ) are unlikely to be of much interest in the strictly theoretical studies of the earth's core.

A few test cases computed suggest also that the refinement is of some importance in conjugate point experiments in geophysics. The point geomagnetically conjugate to $80^{\circ} \mathrm{N}$, $180^{\circ} \mathrm{E}$, using U.S. coefficients for 1955 at ground level, was at latitude $61.44^{\circ} \mathrm{S}, 136.25^{\circ} \mathrm{E}$, or $61.36^{\circ} \mathrm{S}, 136.16^{\circ} \mathrm{E}$, for the the spherical or spheroidal cases, respectively. This can be compared with $61.05^{\circ} \mathrm{S}, 136.68^{\circ} \mathrm{E}$, using U.S. coefficients for 1960 for the spherical case. Consequently, in order that such analytic corrections be of immediate practical interest, it seems necessary to have surface surveys of the geomagnetic field accurate to 100 to 200 gammas, and a need for definition of conjugate point values to within distances of $20 \mathrm{~km}$.

It is interesting to note that a harmonic analysis of the vertical intensity $Z$ of the magnetic field by Jensen and Whitaker (1960) yielded a $g_{0}{ }^{0}$ of $-18.2 \gamma$. This value is an order of magnitude greater than those calculated here as ascribable to the ellipticity of the earth. This $g_{0}{ }^{0}$ is numerically very nearly equal to the average value of $Z$ over the sphere. It can be obtained by integrating $Z$ over the sphere's surface. Such an integral of $Z$ over the earth's surface must vanish. This discrepancy may be related either to random or to systematic errors in the vertical intensity data used in Jensen and Whitaker's analysis. Suppose that the errors in measuring $Z$ are normally distributed, and the data are taken on a $10^{\circ} \times 10^{\circ}$ grid on the earth's surface ( 614 data points). If each measurement of $Z$ were associated with a single small area and all such areas were equal, the average of all possible measurements of $Z$ would be zero. The probable error of the distribution of means of samples taken from such a set of all possible measurements of $Z$ is about $(1 / \sqrt{N})$ times the probable error of a single measurement, where $N$ is the number of measurements in the set. Thus for the $g_{0}{ }^{0}$ term of Jensen and Whitaker to be less than a probable error of $-18.2 \gamma$ over 614 data points, we could infer an absolute probable error of the order of $\pm 450 r$ for a single measurement of the vertical intensity $Z$. Alternatively, we can suppose that the error in $g_{0}{ }^{0}$ in Jensen and Whitaker's analysis is systematic, and confined to say, the antarctic inside a spherical cap of $10^{\circ}$ half angle. In this case, an average systematic error of $2400 r$ over this cap would 
raise the world-wide average of $Z$ by the required $18 \gamma$. Nagata and Oguti (1962) report discrepancies in $Z$ of about $2500 r$ between old charts and corrected charts for the antarctic region based on new data obtained in this region. It appears likely that both systematic and random errors contribute to the value of $g_{0}{ }^{0}$ obtained by Jensen and Whitaker.

These estimates of probable errors are crude, ignoring as they do the nonuniform distribution of data points on the earth's surface, the analytic procedure for computing $g_{0}{ }^{0}$, and a variety of other subtleties. They do however, point up the fact that harmonic analyses of the geomagnetic field can be made to yield information regarding the absolute errors in the data used. The calculation of a magnetic unipole term, independent of any physical hypotheses, yields such information directly. It is advantageous, then, to postpone suppression of the unipole coefficient in harmonic analyses until this measure is obtained. The physical nonexistence of unipoles can then be used to generate another set of coefficients for representing the geomagnetic field.

\section{Acknowledgment}

This paper is the product of research sponsored by the National Aeronautics and Space Administration under Contract NASr-21(05). It does not necessarily represent the views of the National Aeronautics and Space Administration.

\section{References}

Cain, J.C., Discussion at IAGA, XIII General Assembly, IUGG, Berkeley, California, August, 1963.

Chapman, S., and J. Bartels, Geomagnetism, Clarendon Press, Oxford, 1940.

Fanselau, G., and H. Kautzleben, "Die analytische Darstellung des geomagnetischen Feldes," Geofis. Pura e Appl., 41, 33-72, 1958.

Jensen, D.C., and J.C. Cain, “An Interim Geomagnetic Field (Abstract)," J. Geophys. Res., 67, 3568-3569, 1962.

Jensen, D.C., and W.A. Whitaker, "A Spherical Harmonic Analysis of the Geomagnetic Field (Abstract)," J. Geophys, Res., 65, 2500, 1960; (Report) AFSWC, Kirtland Air Force Base, New Mexico, 1960.

Nagata, T., and T. Oguti, "Magnetic Charts of the Period 1958. 5 Corrected for the Antarctic Region and Spherical Harmonic Coefficients of the Revised Geomagnetic Field." J. Geomagnet. Geoelec., 14, 125-131, 1962.

Vestine, E.H., L. Laporte, I. Lange, and W.E. Scott, The Geomagnetic Field, Its Description and Analysis, Carnegic Institution of Washington, Publication 580, Washington D.C., 1947.

Vestine, E.H., W.L. Sibley, J.W. Kern, and J.L. Carlstedt, “Integral and Spherical-Harmonic Analyses of the Geomagnetic Field for 1955. O, Part 1." J. Geomagnet. Geoelec., 15, 47-89, 1963. 